\title{
LAS POLÍTICAS REGIONALES DE CIENCIA, TECNOLOGÍA E INNOVACIÓN EN \\ COLOMBIA: SURGIMIENTO, EVOLUCIÓN Y BALANCE DE LA SITUACIÓN ACTUAL ${ }^{*}$
}

\author{
Edgard MONCAYo JimÉNEZ**
}

\section{Resumen}

Al igual que otros países de América Latina, Colombia comenzó desde la década de los sesenta a construir una política de ciencia, tecnología e innovación (CTI), que ha atravesado por diversas etapas marcadas por diferentes enfoques teóricos y modelos de gobernanza. A la altura de 1990, en el contexto de un fuerte impulso a la descentralización que comprendió diversos campos, comenzó a incorporarse la dimensión regional en dicha política. Desde entonces, y a través de un proceso de ensayo y error, se ha ido constituyendo un corpus de marcos conceptuales, normativos e instrumentales que se ha convertido en un componente infaltable de las políticas de CTI en el país. Con los enfoques de la "territorialización de las políticas públicas” y de la investigación narrativa, este artículo da cuenta del surgimiento, la evolución y la situación actual de las políticas regionales de Cті en Colombia. Especial atención se presta a las tensiones y los dilemas que se están presentando en la actualidad alrededor de

* Este artículo se basa, en parte, en unos textos publicados previamente en la colección de documentos de trabajo del Departamento Nacional de Planeación de Colombia. Específicamente, en los Archivos de Economía 476 y 482 (secciones 1 y 2, y 1 y 3 respectivamente). Al material inicial reelaborado se agregó una introducción con el marco teórico y una sección con las conclusiones pertinentes. En este sentido, el autor agradece los valiosas observaciones y sugerencias de dos árbitros anónimos. Asimismo, agradece al abogado Luis Leguizamón PhD., y al economista Ricardo Mariño, estudiante de maestría, por sus aportes a la preparación de la base de bibliografía comentada en la que se apoyó este trabajo.

** PhD en Ciencias Económicas. Profesor en el doctorado de Modelado de Políticas Públicas, Universidad Jorge Tadeo Lozano, Bogotá (Colombia). [edgardmoncayo@yahoo.com].

Recibido: 12 de abril de 2018 / Modificado: 3 de agosto de 2018 / Aceptado: 16 de agosto de 2018.

Para citar este artículo

Moncayo Jiménez, E. (2018). Las políticas regionales de ciencia, tecnología e innovación en Colombia: surgimiento, evolución y balance de la situación actual. OPERA, 23, 185-208.

DoI: https://doi.org/10.18601/16578651.n23.11 
la integración -a nivel nacional y regional- de las políticas de cті con las de competitividad, que ordenan las normativas recientes.

Palabras clave: política regional, ciencia, tecnología e innovación, evolución, Colombia.

\section{REGIONAL POLICIES ON SCIENCE, TECHNOLOGY AND INNOVATION IN COLOMBIA: EMERGENCE, EVOLUTION AND CURRENT STATUS}

\section{Abstract}

Like other latin american countries, since the decade of 1960 Colombia begin to implement a policy of Science Technology and Innovation (STI) that has undergone several phases reflecting different theoretical approaches and governance models. In the context of the strong push to decentralization comprehending several fields that took place at the beginning of the years of 1990, the regional dimension was introduced to the STI national policy. Since then and through a trial and error process a corpus of conceptual, normative and instrumental approaches regarding this dimension has been progressively built, becoming a essential component of the STI colombian policy. Using the theoretical approaches of "territorialization of public policies" and "narrative research", this article gives an account of the emergence, evolution and present status of the STI colombian policies. Special attention is given to the tensions and dilemmas arising from the convergence at the national and regional levels of the STI and competitiveness policies stablished by recent legislation.
Key words: Regional policies, science technology and innovation, competitiveness, Colombia, emergence, evolution, current status.

\section{INTRODUCCIÓN}

La evolución de las políticas, tecnología e innovación en Colombia ha sido objeto de varios trabajos con diversos periodos de referencia y distintos grados de profundidad en el tratamiento de los temas. Igual cosa ocurre en materia de las políticas de competitividad.

No obstante, en ninguno de estos dos campos se ha estudiado con algún detenimiento y detalle la dimensión regional. En este documento se procura llenar este vacío, analizando el plano regional en el contexto de la coevolución de los dos campos de política pública en cuestión. Se ha considerado pertinente hacer este abordaje, dado que las normativas legales recientes han dispuesto la confluencia de las dos vertientes en un único Sistema Nacional de Competitividad, Ciencia, Tecnología e Innovación.

A los efectos enunciados, se adelanta una reconstrucción de los principales hitos de la regionalización tanto de las políticas de ciencia, tecnología e innovación (СТі), como de las concernientes a la competitividad.

En el periodo abarcado por el estudio (1969-2017) se distinguen cuatro etapas. La que va desde 1969 hasta 1989, en la cual la dimensión regional está ausente en las políticas nacionales de CTI. La de la emergencia o despegue (1990-1999), en la que se incorporan los primeros dispositivos de regionalización 
en el Sistema Nacional de Ciencia y Tecnología organizado en ese periodo, y se llega a la institucionalización de la figura de Sistemas Regionales de Innovación (SRI). Una tercera de 2000 a 2008, en la que las políticas de competitividad se superponen a las de CтI nacional $\mathrm{y}$ regionalmente, $\mathrm{y}$, finalmente, la fase que se extiende desde 2009 hasta 2017, marcada por la convergencia de las políticas de competitividad tanto a escala nacional como regional, por el establecimiento de mecanismos novedosos de financiamiento para los proyectos de сті de origen regio-local y por el arribo a un estadio en el que el plano territorial es un elemento indispensable en las políticas públicas nacionales de сті.

En los estudios sobre las políticas de CTI en Colombia se han empleado diversos enfoques entre los cuales están los de Policy Transfer ${ }^{1}$ y el del Modelo de Sistema Viable (MSv) ${ }^{2}$ (Plata, 2013; Sánchez-Torres y Pérez, 2013). En este trabajo se ha optado por utilizar el marco conceptual de la "territorialización de las políticas públicas" ideado por los teóricos franceses J. Monet (2010), A. Faure (2016) y P. Muller (2010) (independientemente), y aplicado en Colombia por el investigador de esta misma nacionalidad J. F. Jolly (2014).

Esta aproximación proporciona una perspectiva para analizar el tránsito de una política en la que predomina una lógica sectorialvertical (de arriba-abajo) hacia una en la que la lógica territorial-horizontal tiene un espacio importante. En la terminología de esta escuela, estas políticas híbridas adquieren un carácter secto-territorial, que es precisamente lo que ha ocurrido en el campo de la стI en Colombia y en la mayoría de las economías del mundo desarrollado (Laranja, Uyarra, Flanagan, 2008) y de América Latina (Llisterri y Pietrobelli, 2011; González, 2017)³.

La exposición se adelanta, en primer lugar, con un corte diacrónico al hilo de la evolución temporal que van experimentando en el periodo de referencia las iniciativas de regionalización de las políticas de Сті; y en segunda instancia, con un enfoque sincrónico que hace un balance de la situación actual distinguiendo cuatro ejes: planeación y direccionamiento estratégico; convergencia entre Сті у competitividad en el plano territorial; institucionalidad; financiación pública de los proyectos originados en los SRI.

En cada uno de los ejes mencionados se identifican las debilidades y fortalezas, prestando especial atención a las fallas de coordinación entre los niveles nacional y regional, y al interior de los SRI. Cabe anotar que está por fuera del alcance de este trabajo el efecto práctico de los esfuerzos por regionalizar la política nacio-

\footnotetext{
1 Según estas contribuciones, las formas institucionales e instrumentales de la política de CTI en América Latina fueron, con frecuencia, el resultado de la influencia de los países avanzados y de los organismos multilaterales (OECD, BID, OEA, etc.) y, en ocasiones, de la simple copia mimética.

2 Este modelo considera que las condiciones de viabilidad y permanencia de un sistema son determinadas por la interacción entre cinco subsistemas: acción, coordinación, control, inteligencia y política.

3 En este trabajo se describe el proceso de "federalización" -como lo llama la autora- de la política de CTI en Argentina, con un enfoque muy similar al utilizado en el presente documento.
} 
nal de CтI en las capacidades y el desempeño de las entidades subnacionales de este campo.

La reconstrucción histórica de la evolución normativa e institucional del proceso de regionalización de las políticas de CтI plasmada en el texto, también se apoya en las contribuciones de la investigación narrativa aplicada a la administración pública, las cuales valoran esta aproximación como un medio válido de generación del conocimiento (Plata, 2013, p. 70).

Además de esta introducción, el documento tiene otras tres secciones. La segunda y la tercera contienen, respectivamente, los análisis diacrónico y sincrónico aludidos arriba; y en la cuarta se presentan las conclusiones.

\section{EVOLUCIÓN DE LA DIMENSIÓN REGIONAL DE LAS POLÍTICAS DE CIENCIA, TECNOLOGÍA E INNOVACIÓN EN COLOMBIA}

\section{La emergencia de las políticas de ciencia tecnología e innovación ( $\mathrm{CTI}$ ) en Colombia (1960-1989)}

Los autores que se han ocupado de historiografiar la evolución de las políticas de сті en el país coinciden en señalar que las primeras manifestaciones de políticas de tal contenido se produjeron en la década de los sesenta ${ }^{4}$.
Las medidas gubernamentales sobre CTI adoptadas con anterioridad a dicha década se habían limitado a algunos programas y proyectos de investigación científica en los campos de la agricultura y la salud principalmente. También se fundaron instituciones como el Instituto de Fomento Industrial (IFI) en 1940, el Instituto Colombiano de Crédito Educativo y Estudios Técnicos en el Exterior (Icetex) en 1960, el Servicio Nacional de Aprendizaje (Sena) en 1957 y el Instituto de Asuntos Nucleares (IAN) (1959), para desempeñar funciones explícita o implícitamente relacionadas con actividades de CтI.

A principios de la década de los sesenta, Colombia entró en la ola de creación de los "INIAS" con la fundación del Instituto Colombiano Agropecuario (ICA) (1962), que adoptó el mismo modelo utilizado por Argentina (1956), y después en casi todos los países continentales de América Latina.

En 1967, en el marco de una pieza de la mayor trascendencia en la legislación económica colombiana (el Decreto Ley 444/67) se estableció un régimen selectivo a la inversión extranjera y una política de regulación de la compra de tecnología internacional ${ }^{6}$.

El hecho que marcó definitivamente el inicio de las políticas de CTI en Colombia fue la creación del Fondo de Investigaciones Científicas y Proyectos Especiales (Colciencias)

\footnotetext{
4 Para este trabajo se han consultado los siguientes: Villaveces y Forero (2007), Miranda (2014), Plata (2013), Nupia (2014a), Nupia (2014b) y Jaramillo, Villaveces y Cantor (2014).

5 Los institutos de investigación tecnológica agropecuaria que siguieron el modelo del Instituto Nacional de Investigación Agropecuaria (INIA), adoptado por Argentina (Villaveces y Forero, 2007, p. 108).

6 Estos principios se extendieron después, en los años setenta, a los países del Grupo Andino a través de las normativas del Acuerdo de Cartagena, suscrito en 1969.
} 
en 1968 (Decreto 2829 de 1968) ${ }^{7}$. Desde ese momento y hasta la fecha, esta institución se convirtió en el actor central de los esfuerzos que se han realizado en el país para consolidar una política y un sistema nacional de CTI.

Con posterioridad a la creación de Colciencias, y hasta finales de la década de los ochenta, en la arena de las políticas de CTI en Colombia se produjeron diversos procesos de búsqueda de conocimiento, de aprendizaje, de regulación, de diversificación de actores, de legitimación de políticas y de implementación de medidas y proyectos. Entre estos sobresalen:

- La influencia teórica y metodológica de organismos como la Organización de los Estados Americanos (oeA), la Unesco, el Programa de las Naciones Unidas para el Desarrollo (PNUD), la Comisión Económica para América Latina y el Caribe (CEPAL) y la Junta del Acuerdo de Cartagena ${ }^{8}$.

- Activa participación de Colciencias, y otras agencias gubernamentales, principalmente el Instituto Colombiano de Comercio Exterior (Incomex), el Departamento Nacional de Planeación (DNP), y el Banco de la República, en la conformación de una política andina de desarrollo tecnológico que comprendía la transferencia de tecnología, el pago de regalías y programas conjuntos de tecnología para sectores productivos prioritarios (Decisiones 24, 84 y 85 del Acuerdo de Cartagena) (Jaramillo, Villaveces y Cantor, 2014, p. 203).

- Incorporación de la variable tecnológica en los planes nacionales de desarrollo. El primero de estos instrumentos en el que se logró esta operación fue el Plan de Integración Nacional (PIN), de la Administración Turbay Ayala (1978-1982), seguido del plan "Cambio con equidad" (1982-1986) y, con especial énfasis, en el "Plan de Economía Social (PEs)" durante el gobierno del presidente Barco (19861990).

- Financiamiento por parte del Banco Interamericano de Desarrollo (BID) (préstamos al Gobierno de Colombia, fase I, 1983) a través del cual se comenzó a apoyar la participación de empresas del sector público y privado en actividades de сут.

Hasta aquí, ninguno de los procesos mencionados contemplaba la dimensión regional de las políticas de cTi en el país. Solo a finales de la década de los ochenta se produjo la primera manifestación con la creación de Misión de Ciencia y Tecnología en 1988.

Durante la Administración Lleras Restrepo (1966-1970), en el contexto de una amplia reforma administrativa que modernizó la administración pública del Estado colombiano (Acto Legislativo 01 de 1968). En este periodo también se dio inicio al proceso de descentralización fiscal del país.

8 En estas instituciones, especialmente en las dos últimas, se gestó lo que se ha denominado el "pensamiento latinoamericano sobre ciencia, tecnología y sociedad” (Jaramillo, Villaveces y Cantor, 2014, p. 188). 
Atendiendo a las recomendaciones del Foro Internacional sobre Ciencia y Tecnología celebrado en 1987, el presidente Barco dispuso la creación de la Misión de Ciencia y Tecnología (Decreto 1600/88) ${ }^{9}$ que terminó proponiendo la conformación de un Sistema Nacional de Ciencia y Tecnología ${ }^{10}$, vinculado al Plan Nacional de Desarrollo, en cuyo marco se constituyeran las comisiones regionales de ciencia y tecnología.

Estas comisiones se encargarían de la preparación de planes regionales de ciencia y tecnología, que harían parte de los planes y programas nacionales de esta materia (Misión de Ciencia y Tecnología, 1990, p. 122).

Dicha misión hizo, además, otras recomendaciones, como la de promulgar una Ley de Ciencia y Tecnología, crear la Comisión Nacional de Ciencia y Tecnología, adscribir Colciencias al DNP, aumentar el gasto en investigación y desarrollo (I\&D), y desarrollar programas nacionales de desarrollo tecnológico de carácter sectorial. Varias de estas propuestas fueron acogidas por la Ley 29 de 1990, según se reseña en el siguiente apartado.

\section{La conformación del Sistema Nacional de Ciencia y Tecnología, y la emergencia de la dimensión regional de la política de ciencia y tecnología (1990-1999)}

El inicio de la década de los noventa marca un punto de inflexión de mucha trascendencia en la historia económica y política del país. En 1990, la administración Gaviria adoptó un modelo de desarrollo basado en la internacionalización de la economía ${ }^{11}$, y en 1991 una asamblea constituyente aprobó una nueva Constitución nacional.

En este contexto, y acogiendo las recomendaciones de la Misión de Ciencia y Tecnología, el Congreso de la República adoptó en 1990 una Ley Marco para la Ciencia y Tecnología, que representaba la primera normativa de jerarquía legal (de ley) en esta materia en el país.

El mandato de la Ley 29 de 1990 -cuya vigencia se prolongó por 19 años señalaba que:

Corresponde al Estado promover y orientar el adelanto científico y tecnológico y, por lo mismo, está obligado

9 La misión fue dirigida por el economista Gabriel Misas, profesor de la Universidad Nacional, y realizó su trabajo entre 1988 y 1990 (Miranda, 2014, p. 161).

10 La noción de Sistema Nacional de Innovación fue originalmente una contribución de Cristopher Freeman formulada en 1987, lo cual indica que la transferencia de este concepto a Colombia se realizó muy rápidamente. Según Sagastí, lo mismo ocurrió en otros países de América Latina.

11 La "apertura", como dio en llamarse este nuevo modelo, supuso el desmonte de la estrategia la industrialización basada en la substitución de importaciones (III) que había orientado el desarrollo nacional desde principios del siglo xx. Estas reformas estructurales implicaron, en consecuencia, el cese de las políticas (implícitas, la mayoría) asociadas al modelo IsI que se comentaron arriba. 
a incorporar la ciencia y la tecnología a los planes y programas de desarrollo económico y social del país y a formular planes de ciencia y tecnología tanto para el mediano como para el largo plazo (art. 1).

En desarrollo de estas facultades el Gobierno nacional expidió, entre otros, los Decretos Ley 1767 de 1990 y 585 de 1991. Mediante el primero (Estatuto de Ciencia y Tecnología) se introduce el concepto de Sistema Nacional de Ciencia y Tecnología (SNCT) ${ }^{12}$, en consonancia con las innovaciones institucionales que se estaban haciendo por esa época en otros países de América Latina. Además, dispone la creación de las comisiones regionales de cyT, con el fin de proponer los proyectos a mediano y largo plazo para el desarrollo de la ciencia y la tecnología en las regiones.

Esta fue la primera expresión concreta del proceso de regionalización de las políticas de ciencia y tecnología en el país.

Por su parte, el Decreto Ley 585 de 1991 redefinió la integración del Consejo Nacional de Ciencia y Tecnología (СNCут) y dispuso el diseño de una estrategia de regionalización de ciencia y tecnología que contemplara la elaboración de planes regionales.

De esta forma, se crea en Colombia un Sistema Nacional de Ciencia y Tecnología (змсут) que incorporaba una dimensión regional explícita representada en las comisiones regionales, en una estrategia general de regionalización y en unos planes regionales.

Antes de proceder a conformar las comisiones regionales, el CNCyT (a través del DNP y Colciencias) juzgó conveniente hacer un diagnóstico del estado de la сут en las regiones del país. A este efecto, entre 1992 y 1993 se organizaron seis misiones regionales de сут enmarcadas en las jurisdicciones geográficas correspondientes a los Consejos Regionales de Planificación (Corpes) (Ley 1767 de 1990, art. 23) ${ }^{13}$, esto es: Orinoquia, Amazonia, Costa Atlántica, Centro-Oriente, Nor-occidente y Occidente. Cumplida esta etapa, las siete comisiones regionales de сут comenzaron a funcionar entre 1994 y $1997^{14}$.

Los principios y la institucionalidad del Sistema Nacional de Ciencia y Tecnología (sNсут), incluida su dimensión regional, fueron retomados por la Ley 188 de $1995^{15}$ y por los

12 Definido como "el conjunto de funciones e interrelaciones de las entidades públicas y privadas que adelantan la planificación, fomento, financiación y ejecución de la actividad científica y tecnológica, unificada y regulada por los planes de ciencia y tecnología” (art. 2 del Decreto Ley 1767 de 1990).

13 Con fundamento en la Ley 70 de 1985 y los decretos 3083, 3084, 3085 y 3086 de 1985 y con base a un ejercicio de regionalización adelantado por el DNP (Modelo de jerarquización y categorización de entidades territoriales para efectos de planificación), el país se dividió en las seis regiones mencionadas. En cada una de ellas funcionaba un Consejo Regional de Planificación Económica y Social (Corpes).

14 A las seis inicialmente previstas, se agregó la del Distrito Capital por considerarse que esta jurisdicción debería ser tomada en cuenta como una región (Chaparro, 2000, pp. 22-23).

15 Aprobatoria del Plan Nacional de Desarrollo 1995-1998, “El salto social”. Esta fue la primera vez que se aprobó un plan de desarrollo mediante ley de la República. 
documentos del Consejo Nacional de Política Económica y Social (Conpes) 2739 de 1994 y 2875 de 1996 aprobados durante el gobierno del presidente Samper (1994-1998).

En la primera de estas últimas dos normativas se formula por primera vez una política explícita de $\mathrm{CyT}^{16}$, en el marco de la cual se plantea el propósito de dar "impulso al proceso de descentralización de la capacidad de generación y uso del conocimiento, a través de Programas Regionales de Desarrollo Científico y Tecnológico que se articulen con las infraestructuras existentes y respondan a las necesidades de cada región". A este propósito el Conpes 2739 de 1994 recomendaba la adopción de diversos mecanismos regionales.

Por su parte, el Conpes 2875 de 1996 introdujo el concepto de sistema nacional de innovación como un componente del SNCT, que responde a "un modelo interactivo de creación y aplicación del conocimiento, en el que intervienen los diversos agentes ligados con el desarrollo tecnológico y con su vinculación a la producción, dentro de un proceso de búsqueda permanente de la competitividad sostenible y del mejoramiento en la calidad de vida de la población" (Conpes 2875, 1996, p. 1).

Durante la administración Samper Pizano (1994-1998), además de promulgarse las normas ya reseñadas, se reforzó el concepto de competitividad impulsado desde la administración anterior, con la creación del Consejo Nacional de Competitividad (CNC) ${ }^{17}$. La función de este nuevo organismo era asesorar al Gobierno nacional en temas relacionados con la calidad, la productividad y la competitividad tanto a nivel nacional como regional. El CNC se reemplazó en 1998 (Decreto 2222/98) por la Comisión Mixta de Comercio Exterior, organismo adscrito al Ministerio de Comercio Exterior, encargado de asesorar al presidente de la República en temas muy similares a los que atendía su antecesor ${ }^{18}$. Dicho ministerio lanzó en 1999 la Política Nacional de Competitividad y Productividad (PNCP).

\section{La segunda política nacional de ciencia y tecnología, y el avance de la dimensión regional (2000-2002)}

La dimensión regional de cTi se retoma con fuerza en el documento Conpes 3080 de $2000^{19}$, "Política nacional de ciencia y tecnología 2000-2002", uno de cuyos tres objetivos principales era fortalecer la capacidad del sNсут, ampliando su acción y repercusión en las dinámicas sociales, económicas y académicas del ámbito nacional y regional-local.

Para implementar y poner en marcha este propósito, el documento Conpes en mención

\footnotetext{
16 A similitud de lo que se ha dicho sobre la política industrial, se podría afirmar que en Colombia la CTI fue primero una práctica y una institucionalidad, y después una política.

17 Artículos 1 y 2 del Decreto 2010 de 1994. El cNC estaba adscrito al Departamento Administrativo de la Presidencia de la República.

18 A su vez, el Ministerio de Comercio Exterior sería fusionado en 2002 con el Ministerio de Desarrollo para dar paso al Ministerio de Comercio, Industria y Turismo actual.

19 Aprobado en la Administración Pastrana Arango (1998-2002).
} 
recomienda los siguientes mecanismos e instrumentos: los sistemas regionales de innovación; las estrategias regionales de innovación; la ejecución descentralizada de programas nacionales de сут; las tecnologías apropiadas para ecosistemas estratégicos, y las regiones de menor desarrollo relativo; y la preparación de agendas regionales de сут.

Siguiendo esta última recomendación, entre 2000 y 2001 se elaboraron agendas regionales de сут en las mismas seis regiones en las que venían funcionando las comisiones regionales de сут (Orinoquia, Amazonia, Costa Atlántica, Centro-Oriente, Nor-occidente, Occidente y Distrito Capital) (Miranda, 2014, p. 167).

El documento Conpes 3080 de 2000 también hace referencia al concepto de "promoción de la interacción entre la academia, el sector empresarial y sector público para una más eficiente apropiación social del conocimiento, que permita a su vez identificar vocaciones productivas regionales y/o locales" 20 .

Las comisiones regionales de ciencia y tecnología fueron reemplazadas en 2002 por los consejos departamentales de ciencia y tecnología (СОDECyт) a los cuales se encargó la tarea de elaborar, en coordinación con Colciencias, los planes departamentales de CTI (Acuerdo 004 del сNCут).
En el periodo transcurrido entre 2002 y 2009, año este último en el que se promulga una nueva ley de сут, las políticas de competitividad que habían comenzado a gestarse desde principios de los años noventa ${ }^{21}$, toman un fuerte impulso y, de cierta manera, se superponen con las de CTI en algunas áreas.

Así, en el periodo en mención se promulgan numerosas normativas relacionadas específicamente con la competitividad, incluidas algunas atinentes a la dimensión regional ${ }^{22}$ :

- Decreto 1475 de 2008. Creación de las comisiones regionales de competitividad (CRC), coordinadas por el Ministerio de Comercio, Industria y Turismo (MinCIT), y con el apoyo de la Confederación Colombiana de Cámaras de Comercio (Confecámaras) como representante del sector privado.

- Ley 1253 de 2008, "Por la cual se regula la productividad y la competitividad". Dispone que "corresponde a las entidades territoriales señalar los objetivos, metas, prioridades, políticas y estrategias dirigidas a lograr un aumento de la productividad y mejoramiento de la competitividad en armonía con la Ley de Ciencia, Tecnología e Innovación y la

20 Este concepto es lo que en la corriente del pensamiento latinoamericano sobre CTI se denomina el "Triángulo de Sábato" y en la literatura anglosajona la "Triple hélice" (Triple Helix).

21 Los principales antecedentes de estas políticas son: estudios de competitividad de las seis principales ciudades del país, realizados por la firma Consultora Monitor de Michael Porter (1990-1994); creación del Consejo Nacional de Competitividad (CNC) (1994); y reemplazo del cNC por la Comisión Mixta de Comercio Exterior (1998); y la Política Nacional de Productividad y Competitividad (1999).

22 Para los siguientes apartes se han consultado los siguientes trabajos: Reina y Castro (2013), Cárdenas (2008), y Cárdenas, Escalante e Higuera (2010). 
Política Nacional de Productividad y Competitividad incorporada en el Plan de Desarrollo"23 (art. 40).

- Decreto 3737 de 2008, modifica la Alta Consejería para la Productividad y la Competitividad por la Alta Consejería para la Competitividad y las Regiones (establecida en el Decreto 2828 de 2006).

En el mismo lapso (2002-2008), en cambio, se produjeron solo dos normas rectoras relativas a la regionalización de la cTI, que fueron la Ley 812 de 2003, aprobatoria del Plan Nacional de Desarrollo, "Hacia un Estado comunitario", en la cual se incluye una sección dedicada a сті (Cap. II.5), que a su vez comprende un apartado sobre la dimensión regional (Cap. II.5e); y el Decreto 1475 de 2008 que crea las comisiones regionales de competitividad.

\section{La tercera política nacional de cTı, la reforma del SNCT, y la convergencia de las políticas de competitividad y de innovación en el plano regional (2009-presente)}

En 2009 se promulgó la Ley $1286^{24}$ que contiene, entre otras medidas, la de sustituir el Sistema Nacional de Ciencia y Tecnología por el Sistema Nacional de Ciencia, Tecnología e Innovación.

La dimensión regional es objeto de un tratamiento amplio en la Ley 1286 de $2009^{25}$, que comprende los siguientes aspectos: descentralización a través del empoderamiento de las entidades territoriales en materia de Стт $^{26}$; creación de los Consejos Departamentales de Ciencia, Tecnología e Innovación y financiación de proyectos de сті a través regalías (arts. 26 y 27$)^{27}$.

Los principios y las orientaciones de política de cті consignados en la Ley 1286 de 2009 son recogidos y desarrollados en el Documento Conpes 3582 de 2009 "Política Nacional de Ciencia, Tecnología e Innovación”, que respecto a la dimensión regional (sexta estrategia) plantea lo siguiente:

- Fortalecimiento de los Sistemas Regionales de CтI.

- Integración de las estrategias de regionalización del sNCTI con el Sistema Nacional de Competitividad.

- Establecimiento de una política de clústeres, cadenas de valor, parques tecnológicos u otras formas de aglomeraciones de empresas.

\footnotetext{
23 Se refiere al Plan Nacional de Desarrollo 2006-2010, "Estado Comunitario: desarrollo para todos", de la administración Uribe Vélez (2006-2010).

24 En la segunda administración de Álvaro Uribe Vélez (2002-2006). Para una reseńa más amplia de los antecedentes, contexto y alcance de la Ley 1286 véase Miranda (2014) y Ramírez (2011).

25 En este ámbito, la Ley 1286 atendió varias de las recomendaciones planteadas en el documento "Colombia construye y siembra futuro" de Colciencias (2008), que contiene un tratamiento amplio de la dimensión regional.

26 Estas entidades quedaron con las facultadas para crear unidades regionales de investigación científica e innovación, financiadas con sus fondos regionales.

27 Anticipo del Fondo de cTI del SGR.
} 
- Preparación de estrategias para disminuir las disparidades regionales de cTI y permitir que todas las regiones puedan avanzar en este campo.

- Articulación de las instituciones de orden nacional con las de orden regional.

Las reformas al sNCTi consagradas en la Ley 1286 de 2009 y el Conpes 3582 de 2009 del mismo año, representaron varios avances especialmente en lo que concierne a la institucionalidad, la coordinación interinstitucional y la regionalización de la CTI. No obstante, dejaron sin resolver el problema de la financiación insuficiente del sistema, que ha sido una constante a lo largo de toda su evolución.

Por eso el presidente Santos, en los inicios de su primera administración (2010-2012), impulsó la aprobación en el Congreso de la República del Acto Legislativo 05 de 2011 "por medio del cual se constituye el Sistema General de Regalías" (Miranda, 2014, p. 184) 28 .

El mencionado Acto Legislativo creó el Fondo de Ciencia, Tecnología e Innovación (FCTI), alimentado por el 10\% de las regalías que recauda el fisco nacional.

El FCTI financia "proyectos presentados por los departamentos, municipios y distritos que contribuyan a la producción, uso, integración y apropiación del conocimiento en el aparato productivo y en la sociedad en general, para así impulsar el progreso social, el dinamismo económico, el crecimiento sostenible y mayor prosperidad de toda la población" 29 .

Para su aprobación, estos proyectos pasan por el escrutinio del Órgano Colegiado de Administración y Decisión (OCAD), en el cual tienen asiento el Gobierno nacional representado por tres ministros o sus delegados, un representante del DNP y un representante de Colciencias, institución que además ejerce la secretaría técnica; el nivel departamental, cuya representación está en cabeza de un gobernador por cada una de las instancias de planeación regional, cuatro representantes de las universidades públicas y dos representantes de las universidades privadas ${ }^{30}$.

Desde su puesta en funcionamiento hasta el presente, los recursos del FCTI se han repartido con el mismo esquema distributivo del SGR (común a todos los departamentos), el cual obedece a una fórmula calculada por el Ministerio de Hacienda y Crédito Público con base en los indicadores de necesidades básicas insatisfechas (NBI), tamaño de la población y nivel de empleo (art. 34 de la Ley 1530 de 2012). Este modus operandi fue modificado sustancialmente por el Acto Legislativo 4 de 2017, que dispuso que los proyectos por ser financiados seguirán siendo aprobados por el

\footnotetext{
28 Por medio de esta reforma (reglamentada por la Ley 1530 de 2012) se pasó de un Fondo Nacional de Regalías y una concentración de los recursos en las zonas productoras, y puertos marítimos y fluviales por donde se transportan los recursos naturales no renovables, a un sistema que distribuye estos recursos a todas las regiones del país.

29 Ley 1530 de 2012, reglamentaria del Acto Legislativo 5 de 2011, el cual quedó plasmado en los artículos 360 y 361 de la Constitución Nacional.

30 El modelo operativo del Fondo de CTI-SGR es distinto al que maneja el Fondo Francisco José de Caldas, administrado por Colciencias, a través del cual se ejecutan los recursos para CTI procedentes del Presupuesto General de la Nación.
} 
OCAD, pero a través "de convocatorias públicas abiertas y competitivas, articuladas con los correspondientes planes de desarrollo" (art. 1, pará. 15).

En el mismo año de la reforma constitucional que creó el Fondo de Ciencia Tecnología e Innovación del Sistema General de Regalías (FCTeI-SGR), se expidió la Ley 1450 de $2011^{31}$ aprobatoria del Plan Nacional de Desarrollo 2010-2014 "Prosperidad para todos", que en su sección IIIc, "Locomotoras para el crecimiento y la generación de empleo", seleccionó cinco sectores como motores de crecimiento de la economía. El primero de ellos era "Nuevos sectores basados en la innovación”, el cual plantea impulsar la transformación productiva de la economía hacia sectores intensivos en conocimiento, mediante el apoyo de una amplia batería de instrumentos para que las empresas lograran convertir sus ideas e invenciones en productos transables en el mercado nacional e internacional (innovaciones).

Con fundamento en la sección diagnóstica del plan "Prosperidad para todos", que reconocía "la dispersión de esfuerzos por parte de diferentes entidades públicas y privadas relacionadas con la competitividad e innovación, con multiplicidad de instituciones que tiene objetivos comunes tanto en lo regional como en lo nacional, pero que actúan de manera aislada" (DNP, 2011, t. I, p. 213); la Ley 1450 de 2011 dispuso una convergencia de esfuerzos entre las políticas de competitividad (Conpes 3527/08), de transformación productiva (Conpes 3484/07) y de información y comunicaciones (TIC).
Por otra parte, frente a la desarticulación y dispersión de esfuerzos en el plano regional, la norma en mención previó que las CRC serían en adelante las instancias encargadas de coordinar la implementación de las diferentes políticas a este nivel. Lo anterior suponía que en el marco de las CRC se articularan las diferentes instancias regionales existentes en esa época: Consejos Departamentales de Ciencia, Tecnología e Innovación (Codecti), comités UniversidadEstado-Empresa, comités de biodiversidad, redes regionales de emprendimiento, consejos regionales de Pyme, consejos ambientales regionales, comités de seguimiento de los convenios de competitividad e instancias regionales promovidas por el Ministerio de Agricultura y Desarrollo Rural (art. 33) (DNP, 2011, t. II).

Este mecanismo de coordinación fue reforzado por el Decreto 1500 de 2012, que modifica la composición del Consejo Nacional de Ciencia y Tecnología, redefiniendo y organizando, además, el Sistema Nacional de Competitividad bajo nuevos lineamientos. Entre estos estaba el cambio del Sistema Administrativo Nacional de Competitividad que pasó a denominarse Sistema Administrativo Nacional de Competitividad e Innovación, bajo la responsabilidad del Ministerio de Comercio, Industria y Turismo, con el apoyo de la Confederación Colombiana de Cámaras de Comercio (Confecámaras). De esta manera, la implementación de la política de CTI en el plano regional quedó bajo la batuta de las comisiones regionales de competitividad.

En 2012 se plasmó una nueva política de competitividad en la Agenda Nacional de

31 Reglamentada por el Decreto 121 de 2014. 
Competitividad integrada por 109 acciones, cuatro de las cuales estaban relacionadas con CTI: a) programa piloto de extensión tecnológica para Pymes; b) fortalecimiento de centros de Investigación y Desarrollo $(\mathrm{I}+\mathrm{D})$; c) desarrollo de capital humano para la innovación; $\mathrm{y}$ d) promoción de la investigación a través de programas de capacitación en instituciones educativas y empresas.

Como se puede apreciar, no había en las acciones de la Agenda de Competitividad relacionadas con CTI elementos que no hubieran sido contemplados antes en las políticas nacionales de cтr. Tampoco había en dicha agenda una consideración sobre el impacto regional de las acciones incluidas en ella. De todas maneras, este instrumento no tuvo mayor desarrollo práctico porque "las acciones no fueron priorizadas, ni tampoco se realizó un seguimiento de las mismas con el presidente de la República, pues la Comisión Nacional no sesionó con la frecuencia esperada en los últimos años" (Gómez y Mitchell, 2016, p. 14).

Con todo, dicho instrumento se retomó en el Plan Nacional de Desarrollo 2014-2018, "Todos por un nuevo país", aprobado por medio de la Ley 1753 de 2015. Este plan se enfocó en once ejes prioritarios, uno de los cuales se denomina "Competitividad e infraestructura estratégicas", que contempla un objetivo encaminado a "contribuir al desarrollo productivo y la solución de los desafíos sociales del país a través de la ciencia, tecnología e innovación”.

La ley en mención dispone la unificación del Sistema Nacional de Ciencia, Tecnología e Innovación con el Sistema Nacional de Competitividad (art. 7), y fortalece el papel articulador de las comisiones regionales de competitividad, marcando así el último estadio de la convergencia que se había venido buscando desde el Conpes 2739 de $1994^{32}$. Una convergencia en donde la CTI queda puesta al servicio de la competitividad y el desarrollo productivo y empresarial ${ }^{33}$.

En lo referente a la dimensión regional, la Ley 1753 de 2015 dispone en su artículo 7 que el DNP, Colciencias y los departamentos estructuren planes y acuerdos estratégicos departamentales de $\mathrm{CTI}^{34}$, a los cuales deben ajustarse los proyectos que se presenten al OCAD del fCт del Sistema General de Regalías.

Desde la promulgación de la ley en mención se han expedido las siguientes normativas:

32 Sobre este tema habían incidido la Organización para la Cooperación y el Desarrollo Económico (oCDE) y analistas como Bitrán, Benavente y Maggi (2011), y Gómez y Mitchell (2014).

33 La unificación de los sistemas respondió a una sugerencia del informe de la oCDE mencionado arriba. Para una visión crítica de esta convergencia véase Zerda (2015).

34 La disposición de que los planes y acuerdos tengan carácter departamental contradice el PND 2014-2018 que propone, en cambio, estrategias diferenciadas para seis regiones del país, entre las que están las relacionadas con desarrollo productivo, competitividad e innovación. Las regiones propuestas son: Caribe; Eje Cafetero y Antioquia, Centro-Oriente y Bogotá D.C., Pacífico, Llanos y Centro-Sur-Amazonia. Con esta, ya se cuentan ocho propuestas para la regionalización en Colombia, siendo la primera la que se formuló en el primer plan de la administración Lleras Restrepo (1966-1970). De ellas, la única que ha tenido desarrollos prácticos es la de los Corpes, mencionada arriba. 
- Conpes 3866 de 2016. Política Nacional de Desarrollo Productivo. Este documento está basado en una concepción integradora de las políticas de desarrollo productivo, competitividad, y ciencia, tecnología e innovación. En el marco de la articulación que busca dicho documento, se prevé que los departamentos definan agendas integradas de competitividad, ciencia, tecnología e innovación.

- Decreto 849 de 2016. Que define a Colciencias como "organismo principal de la administración pública, rector del sector y del Sistema Nacional de Ciencia, Tecnología e Innovación (sNCTI), encargado de diseñar, formular, orientar, articular, dirigir, combinar, ejecutar e implementar la política de Estado en la materia, en concordancia con los planes y programas de desarrollo". Este decreto también modifica la estructura interna de Colciencias.

- Decreto 584 de 2017. Que reglamenta los Codecti, y Decreto 293 de 2017 (reglamentario del art. 7 de la Ley 1753) relativo a los planes y acuerdos estratégicos departamentales en ciencia, tecnología e innovación (PAED).

\section{DESCRIPCIÓN ANALÍTICA DEL ESQUEMA NORMATIVO Y ORGANIZACIONAL ACTUAL}

Para desarrollar esta sección se procederá primero a sintetizar la evolución de la proyección regional de las políticas de cTi descrita en los apartados anteriores, para luego concluir con la descripción de la situación actual. Al pri- mero de estos efectos, los dispositivos descritos en las cuatro secciones anteriores se agruparán en cuatro ejes: planeación y direccionamiento estratégico, convergencia entre cTі y competitividad en el plano regional, institucionalidad, y descentralización del gasto público en CTI.

\section{Planeación y direccionamiento estratégico}

Con antelación a la conformación del Sistema Nacional de Innovación (Ley 29/91 y Decretos Ley $1767 / 90$ y 585/91) y a la aprobación del documento Conpes 2739 de 1994, en el que se formula la primera política expresa de Сті, las políticas de сті en Colombia no habían contemplado la dimensión regional.

A partir de dichos hitos, y a través de sucesivas aproximaciones, la proyección regional de las políticas de сті se ha ido afirmando progresivamente hasta constituirse en un componente infaltable de las mismas. Son de destacar en tal sentido los siguientes elementos: la incorporación del enfoque regional en las políticas nacionales de CTI, la elaboración de planes y agendas regionales de CтI, la inclusión de la promoción de la cTi en los planes territoriales de desarrollo, la adopción del concepto de sistema regional de innovación (SRI), y el empoderamiento de las entidades territoriales para crear unidades regionales de investigación científica e innovación, financiadas con sus propios recursos.

\section{Convergencia de las políticas de CTI y de competitividad en el plano regional}

Estas dos vertientes de política pública se originaron en la década de los noventa, y 
sus correspondientes normativas tomaron contacto en varios momentos de la evolución, para confluir plenamente en virtud de la Ley 1753 de 2015. Por medio de esta norma, se creó el Sistema Nacional de Competitividad, Ciencia, Tecnología e Innovación (sNCCTI), que engloba las políticas de estos dos campos, tanto a nivel nacional como regional (art. 186).

En el lenguaje utilizado por los documentos Conpes, el sNCCTI se concibe como un "espacio de intersección entre el sncyT y el sector productivo", lo cual implica que la "investigación y la innovación en Colombia se orientan cada vez hacia la competitividad del sector productivo".

En el ámbito regional, la Ley 1753/15 (art. 186) dispone que los Codecti y todas las demás instancias regionales de promoción del desarrollo productivo, empresarial y ambiental deben integrarse a las Comisiones Regionales de Competitividad, que serán la única instancia de interlocución regional con el Gobierno nacional.

Un comité de regionalización ad hoc, que funciona en el Ministerio de Comercio, Industria y Turismo, bajo las directivas de la Alta Consejería Presidencial para la Competitividad y la Productividad de la Presidencia de la República, y del cual hace parte Colciencias, está preparando el decreto reglamentario del artículo 186 de la Ley 1753/15.

\section{Institucionalidad}

Después de la presentación de los informes de las seis misiones regionales organizadas por el DNP y Colciencias entre 1992 y 1993 , con el encargo de hacer el diagnóstico del estado de la CTI en sus respectivas jurisdicciones y hacer las recomendaciones pertinentes ${ }^{35}$, las Comisiones Regionales de Ciencia y Tecnología (СRсут) -previstas en el Decreto-Ley 585/91- comenzaron a funcionar entre 1994 y 1996 .

Las comisiones creadas al inicio fueron: Orinoquia, Armenia, Costa Atlántica, CentroOriente, Nor-occidente y Occidente. A estas se agregó posteriormente la del Distrito Capital en 1997.

Las CRCYT tenían un representante en el Consejo Nacional de Ciencia y Tecnología (CNCT) y en el Consejo de Programas de Colciencias; y conformaron un Comité para el desarrollo de la estrategia de regionalización con la misión de lograr una articulación entre sí, y entre ellas y el sistema nacional. Su misión era el fomento y la difusión de políticas y oportunidades de сут en las regiones; y ser los interlocutores de las regiones con el sistema nacional y con Colciencias (Villaveces y Banguero, 1998, p. 345).

Según Villaveces y Forero (2007), el obstáculo principal con el que tropezaron las CRCyT consistió en la demora que tuvo la Comisión de Ordenamiento Territorial, creada en la Asamblea Nacional Constituyente de 1990, para presentar el proyecto de la Ley que habría de "establecer la distribución de competencias entre la nación y las entidades territoriales" (art. 248 de la Constitución Nacional de 1991). Esta comisión vino a presentar

35 Los informes de las misiones se recogieron en un libro publicado por Colciencias en 1998. 
su proyecto en 1993, pero la iniciativa no hizo tránsito en el Congreso $\mathrm{Nacional}^{36}$.

En estas circunstancias, el Consejo Nacional de Ciencia y Tecnología (CNCT), tomando en cuenta que: "En el actual ordenamiento territorial del estado colombiano y hasta tanto se apruebe la ley Orgánica de Ordenamiento Territorial con el consecuente impulso a la conformación "de las regiones", resolvió reconocer como comisiones regionales de сут, a los consejos, comités o comisiones de ciencia y tecnología del orden departamental, creados formalmente por la autoridad territorial competente (Acuerdo 4 del CNCT).

A partir de 2007, los Consejos Departamentales de Ciencia y Tecnología (CODEcyt) se convirtieron el pilar central de la política de regionalización en esta materia, al punto de que llegaron a instalarse en los 33 departamentos del país. Estos tenían el encargo de elaborar, en coordinación con Colciencias, los planes departamentales de CTI.

Los CODEcyt funcionaron hasta 2009, año en el cual la Ley 1286/09 los transformó en CODECTI, en concordancia con el espíritu general de la norma. Los lineamientos de la integración (membrecía) y las funciones de los CODECTI que están vigentes fueron establecidos por el Decreto 584 de 2017.

En la actualidad hay departamentos que aún no cuentan con CODECTI y se encuentran otros en donde estos organismos sí existen pero están inactivos hasta la fecha de redacción de este artículo $^{37}$. Entre los departamentos que tienen un CODECTI operativo se encuentran varios que han implementado el Decreto 584/17 y otros que están en proceso de hacerlo. Este diverso grado de actualización de la normativa de estos organismos también se registra en el caso de los PAED, según la información proporcionada por la Oficina de Coordinación de los CODECTI de Colciencias ${ }^{38}$.

\section{Financiación}

La política de regionalización de la cті ha contado en los últimos años con una importante fuente de financiación representada en el Fondo de Ciencia, Tecnología e Innovación del Sistema General de Regalías. Los criterios de asignación de los recursos de este fondo van a cambiar sustancialmente con la reglamentación del Acto Legislativo 04 de 2017.

Tradicionalmente, los programas de origen departamental de CTi han contado, además, con la financiación de los programas nacionales que ejecuta directamente Colciencias, puesto que si bien el enfoque de esta fuente no es territorial, es evidente que sus beneficiarios están localizados en alguno de los departamentos del país.

El gran desafío es conciliar la racionalidad de eficiencia económica que aconsejaría destinar recursos a las entidades territoriales

\footnotetext{
36 Solo después de numerosos intentos fallidos, el Congreso Nacional expidió en 2011 la Ley 1454 de Ordenamiento Territorial.

37 Este artículo terminó de redactarse el 8 de marzo de 2018.

38 Comunicación de la señora María Victoria Londoño, coordinadora de la oficina de codecti (fechada el 29 de septiembre de 2017).
} 
con mayor capacidad para utilizarlos adecuadamente, ejecutando proyectos de alto impacto, con la de equidad territorial que busca disminuir las brechas interdepartamentales que se han convertido en uno de los elementos infaltables de las políticas de desarrollo del país.

\section{FORTALEZAS Y DEBILIDADES DE LA INSTITUCIONALIDAD DE CTI Y DE COMPETITIVIDAD A NIVEL NACIONAL Y DEPARTAMENTAL}

Como se desprende de la sección anterior, la institucionalidad de las políticas de competitividad y CTI están en un estado de transición. Como se ha explicado suficientemente, la Ley 1753 de 2015, en su artículo 186, dispuso la "integración del Sistema de Ciencia, Tecnología e Innovación para consolidar un único Sistema de Competitividad, Ciencia, Tecnología e Innovación".

La reglamentación de esta norma se está preparando en el marco de un comité interinstitucional bajo la coordinación de la Alta Consejería Presidencial para el Sector Privado y Competitividad que tenía previsto terminar su trabajo antes de finalizar el año $2017^{39}$, pero hasta el momento no lo ha hecho ${ }^{40}$.

Sobre dicha reglamentación, el documento Conpes 3866 de 2016 (Plan Nacional de Desarrollo Productivo) señala que "las prácticas internacionales aconsejan contar con una arquitectura institucional que diferencie las instancias de coordinación estratégicas y las entidades responsables de la formulación y ejecución", y cuente con unos mecanismos de seguimiento y evaluación (p. 99).

\section{Instancias de direccionamiento estratégico}

Actualmente hay dos instancias que tienen la función de asesorar al Gobierno nacional en las grandes orientaciones estratégicas en los ámbitos de competitividad, ciencia, tecnología e innovación.

Por una parte está la Comisión Nacional de Competitividad e Innovación (CNCI), y por otra el Consejo Asesor de Ciencia, Tecnología e Innovación (СACTI). La CNCI está presidida por el presidente de la República ${ }^{41}$, lo cual asegura que los temas que orientan tengan un alto perfil político, y el CACTI se encuentra presidido por el director del Departamento Administrativo de Ciencia, Tecnología e Innovación (Colciencias).

Sería conveniente que el nuevo sNCCTI, previsto en el artículo 186 la Ley 1753 de 2015, conservara el vínculo directo que tiene la CNCI con la Presidencia de la República ${ }^{42}$ con el fin de ir avanzando progresivamente hacia la consolidación de una política de Estado que atesore la experiencia de casi cincuenta ańos acumulada por el país en esta materia y refleje

\footnotetext{
39 Esta fecha está prevista en el Documento Conpes 3866 de 2016, "Política nacional de desarrollo productivo".

$40 \quad$ Hasta el 8 de marzo de 2018.

41 De su membrecía hacen parte cuatro ministros (Decreto 1500 de 2012).

42 Así está previsto en el documento Conpes 3866 de 2016 (línea de acción 20).
} 
con visión prospectiva los elementos que son comunes a las agendas de los diferentes actores del sistema ${ }^{43}$.

\section{Instancias de diseño, implementación y evaluación}

De acuerdo con el Conpes 3866 de 2016:

A pesar de los esfuerzos realizados a la fecha para institucionalizar el $\mathrm{SNCI}^{44} \mathrm{y}$ mejorar la capacidad de articulación entre los diferentes actores que lo integran, incluyendo las CRC, continúa presentándose descoordinación entre el Gobierno nacional y los gobiernos regionales, entre el sector público y el privado, y entre diferentes entidades del orden nacional. En general, se percibe que no hay claridad sobre las responsabilidades de cada actor, incluyendo quién se encarga de coordinar los distintos temas y actores. Tampoco se ha definido quién realiza seguimiento y evaluación de estas políticas y bajo qué lineamientos (p. 66).

Por otra parte, en cuanto las políticas de CTI, en el documento Conpes "Política Nacional de Ciencia, Tecnología e Innovación 2016-2015"45 se leía:

La coexistencia de diseńo, implementación y monitoreo en las instituciones, en especial en el caso del Ministerio de Comercio Industria y Turismo, Sena y Colciencias [...] dificulta el adecuado balance de las políticas, y debilita el monitoreo de las mismas por parte de las instancias encargadas de su formulación [...] es fundamental resolver estos traslapes con un criterio de especialización de los roles de las entidades ejecutoras ( $\mathrm{p}$. 48) (énfasis agregado).

Dado que los problemas aludidos en los diagnósticos transcritos arriba siguen vigentes, es imperativo que la reglamentación que se está preparando sobre el artículo 186 de la Ley 1753 de 2015 se tome debidamente en cuenta, a fin de que no se reproduzcan y amplifiquen como resultado de la fusión del SNCI y el SNCTI.

En este sentido, como se señaló, en el documento Conpes 3866 se solicitó al MinciT y a Colciencias que prepararan, antes de julio de $2017^{46}$, una propuesta de "Política de Estado", en la cual "se diferencien claramente las funciones de formulación, orientación, dirección, coordinación, ejecución e implementación en el marco del nuevo SNCCTI” (p. 99).

\section{Articulación vertical nación-territorios}

En el varias veces mencionado artículo 166 de la Ley 1753 de 2015 se prevé, además de la integración del SNCI con el de SNCTI, lo siguiente:

... las distintas instancias departamentales que promueven agendas de competitividad, productividad, ciencia, tecnología e innovación, tales como los Codecti,

43 Una política de Estado se refiere a los asuntos que se consideran claves para los intereses generales de una nación, y está guiada por un proyecto estratégico de largo plazo independientemente del contexto político existente en un momento dado.

44 Sistema Nacional de Competitividad e Innovación.

45 Versión para discusión del 11 de marzo de 2016, que no fue finalmente aprobada.

46 Al 18 de marzo de 2018, fecha en la que se terminó de escribir este documento, esto no había ocurrido. 
Comités Universidad-Empresa-Estado, Comités de Biodiversidad, Redes Regionales de Emprendimiento, Consejos Regionales de Pyмe, Consejos Ambientales Regionales, Comités de Seguimiento a los Convenios de Competitividad y las demás que sean promovidas por el Gobierno Nacional, deberán integrarse a las Comisiones Regionales de Competitividad en cada departamento, con el propósito de articular sus agendas de trabajo. Corresponderá a cada Comisión Regional de Competitividad ajustar su estructura de manera que garantice la participación de estas instancias. Las Comisiones serán la única instancia de interlocución con el Gobierno $\mathrm{Na}$ cional para la implementación de la Agenda Nacional de Competitividad, Ciencia, Tecnología e Innovación.

De esta manera, las Comisiones Regionales de Competitividad (CRC) pasan a ser el eje articulador entre el Estado central y las jurisdicciones departamentales.

No obstante, como se anotó arriba, Colciencias expidió el Decreto 584 de 2017, según el cual le corresponde a los CODECTI "articular y coordinar a los actores de la cTl a nivel departamental, para lo cual podrá establecer mecanismos e instancias de cooperación y apoyo interinstitucional”.

El alcance de esta norma está en evidente contradicción con el artículo 186 de la Ley 1753 de 2015 que, como se dijo, le asigna a la CRC el papel de coordinar todas las demás entidades departamentales de promoción del desarrollo, incluidos los CODECTI, por supuesto. Este es un tema que se debe aclarar en la reglamentación que se está preparando, pero es de suponer que la Ley, por ser de mayor jerarquía, prevalezca sobre el decreto de Colciencias.
De otra parte, un nuevo instrumento ha entrado al escenario de la Competitividad, Ciencia, Tecnología e Innovación (ССTI) en el plano regional: las Agendas Integradas Departamentales de Competitividad, Ciencia, Tecnología e Innovación (AIDCCTI).

Las AIDCCTI son el resultado de procesos de integración entre los planes y acuerdos estratégicos departamentales (PAED) y los planes regionales de competitividad, y se fundamentan en la línea 15 del documento Conpes 3866 de 2016, que estableció la

... necesidad de formular planes departamentales de competitividad, ciencia, tecnología e innovación, entendiendo que de la heterogeneidad productiva de las regiones se desprende que las estrategias de priorización de apuestas productivas de los departamentos deben responder a los niveles distintos de diversificación, productividad y sofisticación de cada uno de ellos, así como al hecho de que sus estructuras productivas $y$ potencialidades son diferentes entre sí.

Las AIDCCTI también están llamadas a tomar en cuenta las políticas pertinentes del Plan Nacional de Desarrollo, de los planes departamentales y del sNCCTI. Con el apoyo técnico del DNP y Colciencias, en la actualidad hay nueve departamentos que han adoptado su respectiva AIDCCTI; los demás están en proceso de hacerlo con distinto grado de avance.

Con el nuevo andamiaje institucional de las políticas de CCTI comienzan a superarse varias de las debilidades que se habían detectado en el funcionamiento de las $\mathrm{CRC}^{47}$. Entre estas están las siguientes:

\footnotetext{
47 Los siguientes apartados se basan en las evaluaciones de las CRC realizadas por Gómez y Mitchel (2016) y Nupia (2014).
} 
- La desarticulación entre las CRC, los CODECTI y los otros organismos de promoción del desarrollo regional.

- La desalineación entre los planes de competitividad, los de сті, los planes departamentales y los demás instrumentos de planeación del desarrollo territorial.

- La desalineación entre las políticas nacionales de cсті y las de orden territorial.

- El relativo desconocimiento de la heterogeneidad productiva de las regiones del país en la formulación de los planes de desarrollo.

- La falta de interés que mostraban muchas gobernaciones en las CRC, en la medida que estas nada tenían que ver con los coDECTI que sí son del resorte del Ejecutivo departamental.

No obstante, la reglamentación del artículo 186 de la Ley 1753 de 2015 debería atender varios aspectos que aún están por definir:

- La forma en que las políticas nacionales de planeación y de ссті tomarán en cuenta las AIDCCTI.

- El financiamiento de las CRC, que hoy tienen en la mayoría de los departamentos una dependencia excesiva de las cámaras de comercio.

- El poder vinculante que, para efectos prácticos, tendrían las CRC.

Por último, en lo que concierne al plano regional del nuevo mapa institucional de la CCTI, es importante mencionar que el Acto Legislativo 04 , que modificó la modalidad operativa para la asignación de los recursos del Fondo
CTI/SGR, el cual pasó de cupos preasignados por departamentos a ser convocatorias abiertas y competitivas, está también por reglamentarse.

\section{CONCLUSIONES Y APRECIACIONES FINALES}

En el relato presentado en las tres secciones precedentes se mostró cómo en el periodo 1968-2017 las políticas de CTI en Colombia fueron haciendo una transición gradual pero sostenida desde una lógica sectorial o vertical hacia una territorial u horizontal. Esta última implica descentralización y despliegue de la acción pública en el territorio, que en el caso de este estudio constituye la totalidad de los departamentos del país.

En tal sentido, puede afirmarse que la política en cuestión tiene ahora en Colombia un carácter secto-territorial en el sentido de Pierre Muller, mencionado arriba. Si bien dicha transición aún no es completa, lo cierto es que lo territorial es una dimensión de la política decті queel Estado colombiano ha impulsadode manera continua y progresiva desde los inicios de la década de los noventa hasta la actualidad.

En efecto, desde la expedición de la Ley 29 de 1990, el plano regional ha estado presente en todas las piezas mayores de la normatividad sobre CTI -incluidas las reformas constitucionales y los planes nacionales de desarrollo-; y la institucionalidad, el repertorio de instrumentos y las fuentes de financiación están cada vez más permeados de la visión de "territorialidad".

No obstante, cabe señalar que todavía subsisten problemas de coordinación tanto 
entre el nivel nacional y el regional como al interior de este último. Además, hasta el momento, la regionalización de la política de CTI ha corrido por cuenta de la iniciativa y voluntad del Estado central, lo cual revela la necesidad de implementar los conductos de retroalimentación que permitan canalizar hacia el nivel nacional las iniciativas originadas en las regiones.

\section{REFERENCIAS}

Bitrán, E., Benavente, J. M. y Maggi, C. (2011). Bases para una estrategia de innovación y competitividad para Colombia. Recuperado de http://200.25.59.71:8081/jspui/handle/11146/531

Cárdenas, M. J. (2008). Las politicas de competitividad en Colombia. Bogotá, D.C.: Universidad Sergio Arboleda.

Cárdenas, M., Escalante, C. e Higuera, A. (2010). Marco institucional de la Competitividad en Colombia. En H. Gómez y Pineda, Competitividad e Instituciones en Colombia. Balance y desafíos en áreas estratégicas. Bogotá: U. del Rosario, Consejo Privado de Competitividad y CAF.

Chaparro, F. (2000). Instalación del simposio Ciencia, Innovación y Desarrollo Regional. En Morales y Perdomo (eds.), Simposio "Ciencia, Innovación y Desarrollo Regional”, Memorias. Bogotá: Colciencias.

Colciencias (1994). Ciencias y regiones: la construcción de un país, misiones regionales de ciencia y tecnología. Santafé de Bogotá: Colciencias.

Colciencias (2005). Politica de Apropiación Social de la Ciencia, la Tecnología y la Innovación. Bogotá: Colciencias.
Colciencias (2008). Colombia construye y siembra futuro. Politica nacional a la investigación y la innovación. Bogotá: Colciencias.

Consejo Nacional de Política Económica y Social (1970). Documento Conpes 536 - Transferencia de Tecnología. Bogotá: DNP.

Consejo Nacional de Política Económica y Social (1991). Documento Conpes 2540 - Política de Ciencia y Tecnología. Bogotá: DNP.

Consejo Nacional de Política Económica y Social (1994). Documento Conpes 2739 - Politica Nacional de Ciencia y Tecnología: 1994-1998. Bogotá: DNP.

Consejo Nacional de Política Económica y Social (1996). Documento Conpes 2875 - Autorización a la nación para contratar un crédito externo con la banca multilateral para el proyecto de fortalecimiento al sistema nacional de innovación colombiano. Bogotá: DNP.

Consejo Nacional de Política Económica y Social (1996a). Documento Conpes 2848 - Seguimiento a la Politica Nacional de Ciencia y Tecnología. Bogotá: DNP.

Consejo Nacional de Política Económica y Social (2008). Documento Conpes 3533 - Bases de un plan de acción para la adecuación del sistema de propiedad intelectual a la competitividad y productividad nacional 2008-2010. Bogotá: DNP.

Consejo Nacional de Política Económica y Social (2010). Documento Conpes 3668 - Informe de seguimiento a la Politica Nacional de Competitividad y Productividad Ley 1253 de 2008. Bogotá: DNP.

Consejo Nacional de Política Económica y Social (2010). Documento Conpes 3678 - Política de transformación productiva: un modelo de desarrollo sectorial para Colombia. Bogotá: DNP. 
Consejo Nacional de Política Económica y Social (2015). Documento Conpes 3834 - Lineamientos de politica para estimular la inversión privada en ciencia, tecnología e innovación a través de deducciones tributarias. Bogotá: DNP.

Consejo Nacional de Política Económica y Social (2016). Documento Conpes 3866 - Política Nacional de Desarrollo Productivo. Bogotá: DNP.

Consejo Nacional de Política Económica y Social (2016). Documento Conpes Politica Nacional de Ciencia, Tecnología e Innovación 2016-2025 (versión para discusión del 11 de marzo de 2016). Bogotá. DNP.

Cuervo, J. I. (2014). Ensayos sobre politicas públicas II. Bogotá: Universidad Externado de Colombia.

Departamento Nacional de Planeación (2003). Plan Nacional de Desarrollo 2002-2006: hacia un Estado comunitario. Bogotá: Imprenta Nacional de Colombia.

Departamento Nacional de Planeación (2011). Plan Nacional de Desarrollo 2010-2014, "Prosperidad para todos" (t. 1). Bogotá: DNP.

Departamento Nacional de Planeación (2011). Plan Nacional de Desarrollo 2010-2014, "Prosperidad para todos" (t. II). Bogotá: DNP.

Departamento Nacional de Planeación (2015). Plan Nacional de Desarrollo 2014-2018, “Todos por un nuevo pais". Bogotá: DNP.

Faure, A. (2016). Territorios/territorialización. En J. I. Cuervo, J. F. Jolly y D. Soto (eds.), Diccionario de politicas públicas (pp. 597-605). Bogotá: Universidad Externado de Colombia .

Fondo Colombiano de Investigaciones Científicas y Proyectos Especiales Francisco José de Caldas (1991). Ciencia y tecnología para una sociedad abierta. Bogotá: Colciencias - DNP.

Gómez H. J. y D. Mitchell (2014). Innovación y emprendimiento en Colombia: balance, perspectivas y recomendaciones de politica, 2014-2018. Bogotá: Fedesarrollo.

Gómez, G. y Mitchell, D. (2016). Comisiones regionales de competitividad de Colombia: lecciones para su fortalecimiento institucional (Technical Notes). Inter-American Development Bank. Recuperado de http://publications.iadb.org/ handle/11319/7488

González, G. (2017). Federalización de la ciencia y tecnología en Argentina. Una revisión de iniciativas de territorialización y planificación (1999-2017). Revista de Estudios Regionales, 180, 193-225.

Jaramillo, H., Villaveces, J. y Cantor, N. (2014). El papel de las ideas en ciencia y tecnología en los primeros ańos de Colciencias. Documentos de trabajo 12035. Bogotá: Universidad del Rosario. Recuperado de https://ideas.repec.org/p/ $\mathrm{col} / 000092 / 012035 . \mathrm{html}$

Jolly, J. F. (2014). Territorialidad y territorialización de las políticas públicas: hacia una nueva propuesta de esquema para el análisis de las políticas públicas en el territorio. En J. J. Cuervo (ed.), Ensayos sobre Políticas Públicas II (pp. 275-395). Bogotá: Universidad Externado de Colombia.

Laranja, M., Uyarra, B. R. y Flanagan, K. (2008). Policies for science, tecnology and Innovation: Translating rationales into regional policies in multi-level Setting. Research Policy, 37 (5), 827-835.

Llisterri, J. J. y Pietrobelli, C. (eds.) (2011). Los sistemas regionales de innovación en América Latina, BID. Recuperado de http://190.242.114.8:808/jspvi/ handle/11146/190

Malaver, F. y Vargas, M. (2005). Políticas y avances en la Colombia 1990-2005. Cuadernos de Administración, 18 (30).

Martínez, A., Núñez, J., Castro, F., Gordillo, A., Roveda, G. y Steiner, R. (2015). Levantamiento y con- 
solidación de la línea de base de las inversiones financiadas con recursos del Fondo de Ciencia, Tecnología e Innovación (Informe Final). Bogotá: Fedesarrollo.

Miranda, F. (2014). Reformas de la institucionalidad para la innovación: el caso colombiano. En G. Rivas y S. Rovira (eds.) (2014). Nuevas instituciones para la innovación: prácticas y experiencias en América Latina. Santiago de Chile: Cepal.

Misión de Ciencia y Tecnología (Colombia) (1990). Programa de desarrollo cientifico y tecnológico para Colombia. Bogotá.

Misión de Ciencia, Educación y Desarrollo (Colombia) (1996). Colombia: al filo de la oportunidad. Bogotá: Tercer Mundo Editores. Recuperado de http://www.icesi.net.co/investigaciones_publicaciones/images/pdf/colombia_filo_de_la_oportunidad.pdf

Monnet, J. (2010). Le territoire réticulaire. Revista Antrophos. Huellas del conocimiento, 227, 91-104.

Muller, P. (2010). Las politicas públicas (3 ed. en español). Bogotá: Universidad Externado de Colombia.

Nupia, O. (2013). Colciencias y su papel en la transferencia del modelo internacional de política científica. En M. Salazar et al. (eds.), Colciencias cuarenta años: entre la legitimidad, la normalidad y la práctica (pp. 121-177). Bogotá: OcyT.

Nupia, C. (2014a). La politica cientifica y tecnológica en Colombia, 1968-1991: transferencia y aprendizaje a partir de modelos internacionales. Medellín: Editorial Universidad de Antioquia.

Nupia, C. (2014b). Origen de la política científica y tecnológica en Colombia. Colciencias y su papel en la transferencia del modelo internacional de política científica. En M. Salazar et al. (eds.), Colciencias cuarenta años: entre la legitimidad, la normatividad y la práctica. Bogotá: Observatorio de Ciencia y Tecnología.
Nupia, O. (2014c). Implementación de las políticas de desarrollo productivo en Colombia: una visión desde las regiones. Documento CEDE, 24.

Organisation for Economic Co-operation and Development (OECD) (2014). OECD Reviews of Innovation Policy: Colombia 2014. Paris: Oecd Publishing. http://dx.doi.org/10.1787/9789264204638-en

Plata, J. (2013). Aprendizajes organizacionales y retos en las sociedades del conocimiento. En M. Salazar et al. (eds.), Colciencias cuarenta años: entre la legitimidad, la normalidad y la práctica (pp. 64-119). Bogotá: Observatorio de Ciencia y Tecnología Ocyt.

Ramírez, M. L. (2011). Innovación: elemento indispensable para una politica industrial de nueva generación. Bogotá: Coalición para la Promoción de la Industria Colombiana.

Reina, M. y Castro, F. (2013). 20 años de politicas de Competitividad en Colombia. Bogotá: Fedesarrollo.

Rivas, G. y Rovira, S. (eds.) (2014). Nuevas instituciones para la innovación: prácticas y experiencias en América Latina. Recuperado de http://www. cepal.org/es/publicaciones/36797-nuevas-instituciones-la-innovacion-practicas-experienciasamerica-latina

Salazar, M. et al. (eds.) (2013). Colciencias cuarenta años: entre la legitimidad, la normatividad y la práctica. Bogotá: Observatorio de Ciencia y Tecnología.

Sánchez-Torres, J. M. y Pérez P. (2013). El trasegar de Colciencias en la promoción de la innovación. En Salazar, M. et al. (eds.). Colciencias cuarenta años: entre la legitimidad, la normalidad y la práctica (pp. 418-471) Bogotá: Observatorio de Ciencia y Tecnología.

SNCCTI (2017). Sistema Nacional de Competitividad, Ciencia, Tecnología e Innovación. Recuperado de http://www.colombiacompetitiva.gov.co/ Paginas/SNCEI.aspx 
Villaveces, J. L. y Banquero, H. (2000). Institucionalidad de la ciencia y tecnologías. En Morales y Perdomo (eds.). Simposio "Ciencia, Innovación y Desarrollo Regional”. Memorias. Bogotá: Colciencias.

Villaveces, J. L. y Forero, C. (2007). Cincuenta años de ciencia en Colombia: 1955-2005. En Fundación Alejandro Ángel Escobar, J. L. Villaveces y C. Forero (eds.), Fundación Alejandro Ángel Escobar: 50 años. Bogotá: Fundación Alejandro Ángel Escobar.
Zerda, A. (2015). Ciencia y tecnología en el Plan Nacional de Desarrollo 2014-2018, "Todos por un nuevo país" (Science and Technology in the National Development Plan 2014-2018: "Everyone for a New Country"). SSRN Scholarly Paper ID 2616549. Rochester, NY: Social Science Research Network. Recuperado de https:// papers.ssrn.com/abstract $=2616549$ 